\title{
Theoretical study of infrared and Raman spectra of hydrated magnesium sulfate salts
}

\author{
Galina M. Chaban, Winifred M. Huo, Timothy J. Lee \\ NASA Ames Research Center, Mail Stop T27B-1, Moffett Field, CA 94035-1000
}

\begin{abstract}
Harmonic and anharmonic vibrational frequencies, as well as infrared and Raman intensities, are calculated for $\mathrm{MgSO}_{4} \cdot \mathrm{nH}_{2} \mathrm{O}(\mathrm{n}=1-3)$. Electronic structure theory at the second order Møller-Plesset perturbation theory (MP2) level with a triple-zeta + polarization (TZP) basis set is used to determine the geometry, properties, and vibrational spectra of pure and hydrated $\mathrm{MgSO}_{4}$ salts. The direct vibrational self-consistent field (VSCF) method and its correlation corrected (CC-VSCF) extension are used to determine anharmonic corrections to vibrational frequencies and intensities for the pure $\mathrm{MgSO}_{4}$ and its complex with one water molecule. Very significant differences are found between vibrational spectra of water molecules in complexes with $\mathrm{MgSO}_{4}$ and pure water. Some of the $\mathrm{O}-\mathrm{H}$ stretching frequencies are shifted to the red very significantly (by up to $1500-2000 \mathrm{~cm}^{-1}$ ) upon complexation with magnesium sulfate. They should be observed between 1700 and 3000 $\mathrm{cm}^{-1}$, in a region very different from the corresponding $\mathrm{O}-\mathrm{H}$ stretch frequency region of pure water $\left(3700-3800 \mathrm{~cm}^{-1}\right)$. In addition, the $\mathrm{SO}_{2}$ stretching vibrations are found at lower frequency regions than the water vibrations. They can serve as unique identifiers for the presence of sulfate salts. The predicted infrared and Raman spectra should be of valuable help in the design of future missions and analysis of observed data from the ice surface of Jupiter's moon Europa that possibly contains hydrated $\mathrm{MgSO}_{4}$ salts.
\end{abstract}




\section{Introduction}

Recent observations from the Galileo satellite indicate that three of the Jupiter moons, Europa, Ganymede, and Callisto, may have subsurface oceans ${ }^{1-4}$. Because water is an essential ingredient of life, possible existence of the subsurface ocean and the nature of its composition are of great interest to planetary scientists and astrobiologists. Of the three moons, Europa has attracted the most attention. Data from Galileo's near-infrared mapping spectrometer (NIMS) indicate the possibility of hydrated salts on Europa's

surface $^{5-7}$. The data suggested that Europa's icy crust might contain hydrated sulfates of magnesium and sodium. However, the analysis of NIMS spectra is mainly based on the small shifts and broadening of the water absorption spectra in hydrated salts. Thus, the interpretation of NIMS spectra is not straightforward and does not provide a unique assignment of the observed bands ${ }^{7}$. On the other hand, a definitive identification of the existence of hydrated salts on Europa is of great interest to astrobiologists who have ranked Europa only behind Mars as a potential candidate for extraterrestrial life. Laboratory studies of hydrated $\mathrm{MgSO}_{4}$ and $\mathrm{Na}_{2} \mathrm{SO}_{4}$ salt minerals under low-temperature and ultrahigh vacuum conditions similar to those present on the surface of Europa showed that such salts could exist on the surface of Jupiter's satellite for geologic timescales $\mathbf{8}^{\mathbf{8}}$.

In this study, we use ab initio quantum chemistry calculations to obtain accurate vibrational spectra of magnesium sulfate, $\mathrm{MgSO}_{4}$, hydrated with one, two, and three water molecules. Our calculations provide positions and intensities of vibrational bands for both $\mathrm{MgSO}_{4}$ and water molecules in the hydrated complexes that can serve as unique identifiers for the presence of such salts. The results of our theoretical calculations can assist in the analysis and interpretation of the experimentally observed spectra, and in the design of better detectors for future observations of the Jupiter moon surfaces.

\section{Theoretical Approach}

Stationary points on the potential energy surfaces of magnesium sulfate and its complexes with one, two, and three water molecules are located using second-order 
Møller-Plesset perturbation theory (MP2) ${ }^{9}$ with a triple-zeta + polarization (TZP) basis set: Dunning's basis set for $\mathrm{O}$ and $\mathrm{H}$ atoms ${ }^{\mathbf{1 0}}$ and the McLean-Chandler basis set for $\mathrm{Mg}$ and $\mathrm{S}^{\mathbf{1 1}}$. This level of theory is also used to calculate harmonic and anharmonic vibrational frequencies. Stationary points (minima and transition states) are determined by optimization using analytic gradients of MP2/TZP energies. Second derivative (Hessian) matrices are calculated numerically using double differencing of analytical gradients. Diagonalization of the Hessian matrices provides harmonic frequencies and normal mode coordinates. Anharmonic vibrational frequencies are computed for the smaller systems: pure $\mathrm{MgSO}_{4}$ and its complex with one water molecule. Anharmonic corrections are obtained using the vibrational self-consistent field (VSCF) method $\mathbf{1 2 , 1 3}^{\mathbf{1}}$ and its correlation corrected (CCVSCF) extension via second-order perturbation theory ${ }^{\mathbf{1 4}}$. The calculations are performed using the combined ab initio / CC-VSCF approach, where anharmonic vibrational frequencies are computed directly from an ab initio electronic structure program. Such direct techniques that employ ab initio potential energy surfaces have been developed recently 15,16 and have very important advantages over the techniques that use analytical potential functions. They allow the application of anharmonic vibrational spectroscopy methods to any molecular system of moderate size (up to 10-15 atoms), for which analytical potential functions are not available ${ }^{15}$. The VSCF method is used to solve the vibrational Schrödinger equation to obtain vibrational wavefunctions and energies. In the VSCF approximation, the total vibrational wavefunction in mass-weighted normal coordinates is represented as a product of one-dimensional functions (each depending on one normal coordinate), and the resulting one-dimensional VSCF equations are solved selfconsistently. The VSCF energies are further corrected for correlation effects between the vibrational modes using second order perturbation theory (CC-VSCF) ${ }^{\mathbf{1 4}}$. To make the integrals involved in the CC-VSCF calculations more tractable and to reduce the number of electronic structure computations required, a pairwise coupling approximation is used for 
the potential in the normal-mode representation ${ }^{14}$. In this approximation, the potential of the system is represented by the sum of separable (single mode) terms and pair coupling terms, neglecting triple coupling of normal modes and higher-order interactions. This approximation was shown to work well in a number of previous calculations of hydrogenbonded systems ${ }^{17}$. A grid representation for the potential energy terms is used, where onedimensional separable terms for each normal mode are represented by a grid of 16 points, while pair coupling potential terms for each pair of normal modes is represented by a $16 \times 16$ square grid. The potential energy values are computed on less dense $(8 \times 8)$ grids directly from the ab initio program and subsequently interpolated into $16 \times 16$ grids using polynomial interpolation. The number of potential energy points that needs to be computed in the above algorithm is equal to $N_{\text {points }}=N_{\text {mode }} * N_{\text {grid }}+\frac{N_{\text {mode }} *\left(N_{\text {mode }}-1\right)}{2} * N_{\text {grid }}^{2}$ where $\mathrm{N}_{\text {mode }}$ is the number of normal modes of the system; $\mathrm{N}_{\text {grid }}$ is the number of grid points in one dimension. The same MP2/TZP ab initio level used to obtain equilibrium structures and harmonic normal modes is also used to compute potential energies on grids. Full details of the CC-VSCF method combined with an ab initio electronic structure code is given in Ref. $[15,17]$. All calculations in this study are performed using the electronic structure package GAMESS 18 .

Previous studies showed that the MP2/TZP level of theory gives satisfactory results for geometrical structures and spectroscopic properties of different hydrogen-bonded complexes: water clusters ${ }^{17}$, complexes of negative and positive ions with water ${ }^{17}$, and complexes of acids ( $\mathrm{HCl}$ and $\mathrm{HF}$ ) with water $\mathbf{1 9 , 2 0}$. The combined ab initio / CC-VSCF technique that accounts for anharmonicities and couplings between vibrational modes (in conjunction with the MP2/TZP ab initio potentials) was shown to predict anharmonic frequencies for the highest frequency stretching vibrations with the accuracy of $30-50 \mathrm{~cm}^{-1}$ compared with available experimental data ${ }^{17,19,20}$. Though this is not at the level of 
spectroscopic accuracy, it is a substantial improvement over the harmonic level theoretical predictions. Furthermore, even at this level of accuracy theoretical calculations can be used to help analyze observed spectra and predict important spectroscopic features. The method has been shown to have sufficient accuracy even for highly anharmonic hydrogen-bonded systems. Complexes of magnesium sulfate with water considered in this study are expected to be very anharmonic. Reliable prediction of their spectroscopic properties requires methods that go beyond the harmonic approximation. The combined CC-VSCF / ab initio method should give useful predictions for the most important spectroscopic features characteristic for such hydrated magnesium sulfate systems.

\section{Results and Discussion}

\section{A. Equilibrium geometries, energetics, and charge distribution}

The structure of the pure $\mathrm{MgSO}_{4}$ and pure water molecule obtained at the MP2/TZP level of theory are shown in Fig. 1. Also shown are partial charges, obtained using the natural bond orbital (NBO) analysis 21 . The figure shows large positive charges on $\mathrm{S}$ and $\mathrm{Mg}$ atoms. The smallest $1: 1$ complex $\left(\mathrm{MgSO}_{4} \cdot \mathrm{H}_{2} \mathrm{O}\right)$ is found to have two stable isomers, shown in Fig. 2. The one that corresponds to the global minimum has a hydrogen bond between the hydrogen atom of the water molecule and one of the oxygens of $\mathrm{MgSO}_{4}$. The $\mathrm{O}-\mathrm{H}$ bond that participates in this hydrogen bonding is significantly elongated compared to $\mathrm{O}-\mathrm{H}$ distances of the pure water molecule (by $0.086 \AA$ ). One of the $\mathrm{S}=\mathrm{O}$ bonds is also elongated by $0.056 \AA$, while the other $S=O$ bond is slightly shortened (by $0.01 \AA$ ). In addition to this very strong hydrogen bond, there is also a strong electrostatic interaction between $\mathrm{Mg}$ atom and the oxygen of $\mathrm{H}_{2} \mathrm{O}$, also present in the other isomer (Fig. 2b). It is found that the first (hydrogen-bonded) isomer has a slightly smaller dipole moment than the pure $\mathrm{MgSO}_{4}$ molecule (12.15 Db vs. $12.85 \mathrm{Db}$ ), while the second isomer has a much larger dipole moment (17.30 Debye). Binding energies of the two $\mathrm{MgSO}_{4} \cdot \mathrm{H}_{2} \mathrm{O}$ 
complexes, calculated at the MP2/TZP level of ab initio theory, are 42 and $41 \mathrm{kcal} / \mathrm{mol}$. All energetics are summarized in Table 1.

Two isomers are also found for the complex of $\mathrm{MgSO}_{4}$ with two water molecules (Fig.3). The lowest energy isomer has one water molecule that is connected to the $\mathrm{Mg}$ atom only, and the other water molecule that in addition forms a hydrogen bond with an oxygen atom from $\mathrm{MgSO}_{4}$, similar to the hydrogen bond in the complex with one water molecule (Fig. 2a). This isomer has a dipole moment of 13.32 Debye. Its total binding energy is 79 $\mathrm{kcal} / \mathrm{mol}$ or $39.5 \mathrm{kcal} / \mathrm{mol}$ per water molecule. The second isomer has a "bridge" of two water molecules connecting the $\mathrm{Mg}$ atom with one of the oxygens of the $\mathrm{SO}_{4}$ group. This isomer is about $12 \mathrm{kcal} / \mathrm{mol}$ higher in energy than the first (lowest energy) one. The two hydrogen bonds of the "bridge" in the second isomer are also very strong, but the participating $\mathrm{O}-\mathrm{H}$ bond lengths are not as elongated as in the $3 \mathrm{a}$ and $2 \mathrm{a}$ structures.

The two isomers of the complex of $\mathrm{MgSO}_{4}$ with three water molecules are shown in Fig. 4. Again, the lowest energy isomer has only one hydrogen-bonded water' and two "free" water molecules bound only to $\mathrm{Mg}$ atom. The total binding energy of this complex is 109 $\mathrm{kcal} / \mathrm{mol}$ or $36.2 \mathrm{kcal} / \mathrm{mol}$ per water molecule, and its dipole moment is 13.27 Debye. The hydrogen bond in this complex is similar to hydrogen bonds in the lowest energy complexes of $\mathrm{MgSO}_{4}$ with one and two water molecules, but is slightly weaker. This hydrogen bond is the strongest in the 1:1 complex and becomes somewhat weaker as the number of water molecules increases. The second isomer has a two-water "bridge" and a hydrogen-bonded water molecule. It is $4.6 \mathrm{kcal} / \mathrm{mol}$ higher in energy than the other (most stable) isomer and has a smaller dipole moment of 11.30 Debye. The hydrogen bonds of this isomer are not as strong as in other isomers, including the hydrogen bonds of the "bridge", which are weaker than those in the similar bridge of the complex with two water molecules (structure $3 b$ ). 


\section{B. Vibrational spectra}

Harmonic and anharmonic (CC-VSCF) vibrational frequencies as well as infrared and Raman intensities for the isolated water molecule, isolated $\mathrm{MgSO}_{4}$, and the complex $\mathrm{MgSO}_{4} \cdot 1 \mathrm{H}_{2} \mathrm{O}$ obtained in this study are presented in Table 2. Comparison of vibrational frequencies of the water molecule in the 1:1 complex with $\mathrm{MgSO}_{4}$ (where it forms the hydrogen bond with one of the oxygen atoms of $\mathrm{SO}_{4}$ ) with vibrational frequencies of the pure water molecule, shows that the frequency of one of the $\mathrm{O}-\mathrm{H}$ stretching vibrations is reduced by about $1400 \mathrm{~cm}^{-1}$ at the harmonic level (down to $2450 \mathrm{~cm}^{-1}$ ). When anharmonic corrections are included, this frequency shift due to complexation of $\mathrm{H}_{2} \mathrm{O}$ with $\mathrm{MgSO}_{4}$ is even more significant, almost $2000 \mathrm{~cm}^{-1}$. The CC-VSCF frequency for this $\mathrm{O}-\mathrm{H}$ stretch is $1642 \mathrm{~cm}^{-1}$, which is in the region of water bending frequencies. However, its infrared and Raman intensities are very high, much higher than the corresponding intensities of the water bending vibrations. Therefore, this unusually red-shifted $\mathrm{O}-\mathrm{H}$ stretching vibration should be observable and easily distinguishable from bending vibrations.

Since the anharmonic correction for this $\mathrm{O}-\mathrm{H}$ stretch is unusually large (about $800 \mathrm{~cm}^{-}$

1), we have checked the stability of this result by computing more dense grids for the pair coupling potentials of this mode with all other normal modes of the complex. $16 \times 16$ point grids were computed and interpolated into more dense $32 \times 32$ point grids. This changed the vibrational frequencies of the complex by only a few $\mathrm{cm}^{-1}$, including the value of the redshifted $\mathrm{O}-\mathrm{H}$ stretch that changed only slightly, from 1642 to $1639 \mathrm{~cm}^{-1}$. This shows that the calculated frequency for this unusually red-shifted and anharmonic vibration is not a numerical artifact, but a stable result.

It can be also seen from Table 2 that one of the $\mathrm{S}=\mathrm{O}$ stretching vibrations of $\mathrm{MgSO}_{4}$ is also red-shifted, but to a lesser extent, by about $200 \mathrm{~cm}^{-1}$ upon complexation with one water molecule, and its infrared intensity is significantly enhanced. In the second isomer of 
$\mathrm{MgSO}_{4} \cdot \mathrm{H}_{2} \mathrm{O}$ (Fig. 2b), the water molecule does not form a hydrogen bond, and its frequencies are not changed as much as in the hydrogen-bonded isomer relative to the isolated water molecule. However, its infrared intensities are also significantly enhanced and therefore should be distinguishable from the pure water vibrations.

The effects found for the complex of $\mathrm{MgSO}_{4}$ with one water molecule are also present in complexes of $\mathrm{MgSO}_{4}$ with two and three water molecules. The $\mathrm{O}-\mathrm{H}$ stretching frequencies of these complexes are significantly shifted to the red compared with the isolated water, but to a somewhat lesser extent than in the $1: 1$ complex. Note that anharmonic corrections were not obtained for the complexes of $\mathrm{MgSO}_{4}$ with two and three molecules, since CC-VSCF calculations for such large systems are very expensive. However, comparison of harmonic frequencies for these systems with those of the $1: 1 \mathrm{MgSO}_{4} \cdot \mathrm{H}_{2} \mathrm{O}$ complex shows that the $\mathrm{O}-\mathrm{H}$ stretching frequencies of the larger complexes are red-shifted to a lesser extent. The red shifted $\mathrm{O}-\mathrm{H}$ stretches of the $\mathrm{MgSO}_{4} \cdot 2 \mathrm{H}_{2} \mathrm{O}$ complexes are located at about 3400,2800 , and $2500 \mathrm{~cm}^{-1}$ at the harmonic level, while those of the $\mathrm{MgSO}_{4} \cdot 3 \mathrm{H}_{2} \mathrm{O}$ complexes are at about $3450,2940,2800$, and $2660 \mathrm{~cm}^{-1}$ (harmonic level). We expect these frequencies to go down significantly when anharmonic corrections are included. In our estimate, the anharmonic corrections should be of the order of several hundreds $\mathrm{cm}^{-1}, 350$ to $600 \mathrm{~cm}^{-1}$ on average. Thus, the red shifted O-H stretches should be observed in the region between 3200 and 2000 $\mathrm{cm}^{-1}$. This region is very different from pure water and water cluster frequency regions: $3600-$ $3800 \mathrm{~cm}^{-1}$ for $\mathrm{O}-\mathrm{H}$ stretches and $1600 \mathrm{~cm}^{-1}$ for $\mathrm{HOH}$ bends. The infrared intensities of these vibrations are about 20-30 times higher than those of the strongest pure water O-H vibrations. Observation of such intense infrared vibrations in the $2000-3200 \mathrm{~cm}^{-1}$ region should be indicative of the presence of hydrated salts. 
In addition to $\mathrm{O}-\mathrm{H}$ stretches, water bending vibrations also change upon complexation with magnesium sulfate. Their frequencies are somewhat higher (blue shifted) in the

complexes (up to $1660-1710 \mathrm{~cm}^{-1}$ ) relative to the pure water molecule (about $1600 \mathrm{~cm}^{-1}$ ), and they also have larger infrared intensities (see Tables 2-4).

Unlike O-H stretches for water molecules, the vibrational frequencies of the $\mathrm{MgSO}_{4}$ fragment are found to be nearly harmonic, except for the $\mathrm{S}-\mathrm{O}$ stretching vibration of the $\mathrm{S}=\mathrm{O}$ bond participating in the hydrogen bonding with water. This $\mathrm{S}=\mathrm{O}$ stretching vibration goes down from 1009 to $935 \mathrm{~cm}^{-1}$ in the $\mathrm{MgSO}_{4} \cdot \mathrm{H}_{2} \mathrm{O}$ complex when anharmonic corrections are included. The highest $S=O$ stretching frequency is located around $1310-1320 \mathrm{~cm}^{-1}$, and its position almost does not change in the different complexes. This vibration, and the other $\mathrm{S}=\mathrm{O}$ stretch in the region of $1000 \mathrm{~cm}^{-1}$, have very large intensities and can serve as unique fingerprints of the presence of sulfate fragments.

\section{Conclusions}

Geometrical structure, properties, and vibrational spectra of pure and hydrated $\mathrm{MgSO}_{4}$ salts are calculated using electronic structure theory at the second order MøllerPlesset perturbation theory (MP2) level with a triple-zeta + polarization (TZP) basis set. For smaller systems (pure $\mathrm{MgSO}_{4}$ and its complex with one water molecule) anharmonic corrections to vibrational frequencies are computed using the correlation corrected vibrational self-consistent field (CC-VSCF) method.

Very significant differences are found between vibrational spectra of water molecules in complexes with $\mathrm{MgSO}_{4}$ and pure water. Some of the water $\mathrm{O}-\mathrm{H}$ stretching frequencies are shifted to the red very significantly upon complexation with magnesium sulfate, and their infrared intensities are greatly enhanced. This effect is due to very strong hydrogen bonds formed between water molecules and the oxygen atoms of $\mathrm{MgSO}_{4}$. The red shift is largest 
for the smallest $1: 1 \mathrm{MgSO}_{4} \cdot \mathrm{H}_{2} \mathrm{O}$ complex, where the $\mathrm{O}-\mathrm{H}$ stretching vibration is shifted by almost $2000 \mathrm{~cm}^{-1}$ and is located at about $1640 \mathrm{~cm}^{-1}$. This vibration should be easily distinguishable from $\mathrm{HOH}$ bending vibrations since it is calculated to have a very high infrared intensity (almost 20 times that of the $\mathrm{HOH}$ bending in the pure water). Strongly red shifted $\mathrm{O}-\mathrm{H}$ stretches are also present in complexes of $\mathrm{MgSO}_{4}$ with two and three water molecules, but this effect becomes less significant as the number of water molecules around $\mathrm{MgSO}_{4}$ increases. However, even for the situation where $\mathrm{MgSO}_{4}$ has high number of hydration waters, the hydrogen bonded $\mathrm{O}-\mathrm{H}$ stretching frequency should be observed in the region between 2000 and $3200 \mathrm{~cm}^{-1}$, which differs significantly from pure water and water cluster frequency regions $\left(3600-3800 \mathrm{~cm}^{-1}\right)$. Water bending frequencies in complexes may be easier to identify, since they do not change as much as the stretching frequencies. They are found to shift to the blue by up to $100 \mathrm{~cm}^{-1}$ upon complexation with magnesium sulfate. In addition, the $\mathrm{SO}_{2}$ stretching vibrations are found at lower frequency regions $\left(900-1300 \mathrm{~cm}^{-1}\right)$ than the water vibrations. They can serve as unique identifiers for the presence of sulfate salts. The predicted infrared and Raman spectra should be of valuable help in the design and analysis of experimental observations of the icy surface of Jupiter's moon Europa that possibly contains hydrated $\mathrm{MgSO}_{4}$ salts.

For the $1: 1 \mathrm{MgSO}_{4} \cdot \mathrm{H}_{2} \mathrm{O}$ complex, the anharmonic correction of the red-shifted O$\mathrm{H}$ stretch, calculated using CC-VSCF, is very large, about $800 \mathrm{~cm}^{-1}$. Anharmonic corrections of this size have not been obtained previously for neutral hydrogen-bonded complexes. Experimental measurement of this transition will be very useful as a test of our theoretical treatment. 


\section{Acknowledgement}

The research was supported by the Director's Discretionary Fund at NASA Ames Research Center. 


\section{References}

1. Showman, A. P, Malhotra, R. Science, 1999, 286, 77.

2. Kargel, J. S. et al. Icarus, 2000, 148, 226.

3. Ruiz, J. Nature, 2001, 412, 409.

4. McCord, T. B.; Hansen, G. B.; Hibbitts, C. A, Science, 2001, 292, 1523.

5. McCord, T. B. et al. J. of Geophys. Research. 1998, 103, 8603.

6. McCord, T. B. et al. J. of Geophys. Research. 1999, 104, 11827.

7. McCord, T. B.; Hansen, G. B.; Shirley, J. H.; Carlson, R. W. J. of Geophys.

Research. 1999, 104, 27157.

8. McCord, T. B.; Orlando, T. M.; Teeter, G.; Hansen, G. B.; Sieger, M. T.; Petrik, N.

G.; Van Keulen, L. J. of Geophys. Research - Planets. 2001, 106, 3311.

9. J. A. Pople, J. S. Binkley, and R. Seeger, Int. J. Quantum Chem. 1976, 10, 1.

10. T. H. Dunning. J. Chem. Phys. 1971, 55, 716.

11. A. D. McLean, G. S. Chandler. J. Chem. Phys. 1980, 72, 5639.

12. J. M. Bowman. J. Chem. Phys. 1978, 68, 608.

13. R. B. Gerber and M. A. Ratner. Chem. Phys. Lett. 1979, 68, 195.

14. J.-O. Jung and R. B. Gerber, J. Chem. Phys. 1996, 105, 10332.

15. Chaban, G. M.; Jung, J.-O.; Gerber, R. B. J. Chem. Phys. 1999, 111, 1823.

16. Yagi, K.; Taketsugu, T.; Hirao K.; Gordon, M.S. J. Chem. Phys. 2000, 113, 1005.

17. Chaban, G. M.; Jung, J.-O.; Gerber, R. B. J. Phys. Chem. A 2000, 104, 2772.

18. Schmidt, M. W.; Baldridge, K. K.; Boatz, J. A.; Elbert, S. T.; Gordon, M. S.; Jensen, J. H.; Koseki, S.; Matsunaga, N.; Nguyen, K. A.; Su, S.; Windus, T. L.;

Dupuis, M.; Montgomery, J. A. J. Comp. Chem. 1993, 14, 1347.

19. Chaban, G. M.; Gerber, R. B.; Janda, K. C. J. Phys. Chem. A 2001, 105, 8323.

20. Chaban, G. M.; Gerber, R. B.; Spectrochim. Acta A 2002, 58, 887. 
21. A. E. Reed, F. Weinhold, J. Chem. Phys. 1983, 78, 4066; A. E. Reed, R. B. Weinstock, F. Weinhold, J. Chem. Phys. 1985, 83, 735. 
Table 1. Total energies (hartrees) and dipole moments (Debye) of complexes of $\mathrm{MgSO}_{4}$ with water.

\begin{tabular}{|l|r|c|}
\hline & \multicolumn{1}{|c|}{ Total energy } & Dipole moment \\
\hline $\mathrm{MgSO}_{4}$ & -897.611838 & 12.846 \\
\hline $\mathrm{H}_{2} \mathrm{O}$ & -76.274033 & 2.232 \\
\hline $\mathrm{MgSO}_{4} \cdot 1 \mathrm{H}_{2} \mathrm{O}$ isomer a & -973.953258 & 11.661 \\
isomer b & -973.951329 & 17.308 \\
\hline $\mathrm{MgSO}_{4} \cdot 2 \mathrm{H}_{2} \mathrm{O}$ isomer a & -1050.285492 & 13.318 \\
isomer b & -1050.266681 & 13.249 \\
\hline $\mathrm{MgSO}_{4} \cdot 3 \mathrm{H}_{2} \mathrm{O}$ isomer a & -1126.607081 & 13.272 \\
isomer b & -1126.599753 & 11.304 \\
\hline
\end{tabular}


Table 2. Vibrational frequencies $\left(\mathrm{cm}^{-1}\right)$, infrared (Debye $2 / \AA^{2}$.amu) and Raman $\left(\AA^{4} / \mathrm{amu}\right)$ intensities of $\mathrm{MgSO}_{4}, \mathrm{H}_{2} \mathrm{O}$, and $\mathrm{MgSO}_{4} \cdot 1 \mathrm{H}_{2} \mathrm{O}$ complex.

\begin{tabular}{|l|c|c|r|r|}
\hline & \multicolumn{2}{|c|}{ frequencies } & \multicolumn{2}{l|}{ intensities } \\
\hline & harmonic & CC-VSCF & IR & Raman \\
\hline $\mathrm{H}_{2} \mathrm{O}$ & & & & \\
\hline asym. $\mathrm{OH}$ stretch & 4015 & 3797 & 1.19 & 34.99 \\
\hline sym. $\mathrm{OH}$ stretch & 3877 & 3691 & 0.17 & 72.48 \\
\hline $\mathrm{HOH}$ bend & 1603 & 1548 & 1.80 & 6.21 \\
\hline $\mathrm{MgSO}_{4}$ & & & & \\
\hline asym. $\mathrm{S}=\mathrm{O}$ stretch & 1347 & 1326 & 7.01 & 22.13 \\
\hline sym. $\mathrm{S}=\mathrm{O}$ stretch & 1167 & 1153 & 6.33 & 15.67 \\
\hline $\mathrm{MgSO}_{4} \cdot 1 \mathrm{H}_{2} \mathrm{O}$, isomer & & & & \\
\hline free $\mathrm{OH}$ stretch & 3958 & 3802 & 3.16 & 83.49 \\
\hline bonded OH stretch & 2451 & 1642 & 33.97 & 51.36 \\
\hline $\mathrm{HOH}$ bend & 1676 & 1547 & 7.07 & 7.39 \\
\hline free $\mathrm{S}=\mathrm{O}$ stretch & 1333 & 1318 & 8.90 & 11.44 \\
\hline bonded S=O stretch & 1009 & 935 & 10.95 & 13.06 \\
\hline $\mathrm{MgSO}{ }_{4} \cdot 1 \mathrm{H}_{2} \mathrm{O}$, isomer b & & & & \\
\hline asym. OH stretch & 3946 & & 5.41 & 26.45 \\
\hline sym. $\mathrm{OH}$ stretch & 3845 & & 3.17 & 64.97 \\
\hline $\mathrm{HOH}$ bend & 1685 & & 3.36 & 1.03 \\
\hline asym. S=O stretch & 1330 & & 7.61 & 14.50 \\
\hline sym. $\mathrm{S}=\mathrm{O}$ stretch & 1162 & & 6.98 & 14.32 \\
\hline
\end{tabular}


Table 3. Vibrational frequencies $\left(\mathrm{cm}^{-1}\right)$, infrared (Debye $\left.2 / \AA^{2} \cdot \mathrm{amu}\right)$ and Raman $\left(\AA^{4 / \mathrm{amu})}\right.$ intensities of complexes of $\mathrm{MgSO}_{4}$ with two water molecules

\begin{tabular}{|c|c|c|c|}
\hline & \multirow{2}{*}{$\begin{array}{l}\text { harmonic } \\
\text { frequencies }\end{array}$} & \multicolumn{2}{|c|}{ Intensities } \\
\hline & & IR & Raman \\
\hline \multicolumn{4}{|l|}{$\mathrm{MgSO}_{4} \cdot 2 \mathrm{H}_{2} \mathrm{O}$, isomer a } \\
\hline W1: free OH stretch & 3961 & 2.59 & 81.04 \\
\hline bonded $\mathrm{OH}$ stretch & 2499 & 35.30 & 63.47 \\
\hline $\mathrm{HOH}$ bend & 1693 & 5.22 & 8.14 \\
\hline W2: asym. OH stretch & 3964 & 4.78 & 51.83 \\
\hline sym. OH stretch & 3808 & 2.45 & 54.84 \\
\hline $\mathrm{HOH}$ bend & 1655 & 4.32 & 1.39 \\
\hline free $\mathrm{S}=\mathrm{O}$ stretch & 1320 & 9.00 & 9.04 \\
\hline bonded $\mathrm{S}=\mathrm{O}$ stretch & 1020 & 10.80 & 12.62 \\
\hline \multicolumn{4}{|l|}{$\mathrm{MgSO}_{4} \cdot 2 \mathrm{H}_{2} \mathrm{O}$, isomer $\mathrm{b}$} \\
\hline W1: free OH stretch & 3973 & 4.19 & 83.27 \\
\hline bonded $\mathrm{OH}$ stretch & 2807 & 33.38 & 72.44 \\
\hline $\mathrm{HOH}$ bend & 1705 & 4.95 & 4.65 \\
\hline W2: asym. OH stretch & 3949 & 2.83 & 60.03 \\
\hline sym. OH stretch & 3419 & 21.69 & 125.85 \\
\hline $\mathrm{HOH}$ bend & 1667 & 1.61 & 4.17 \\
\hline free $S=O$ stretch & 1337 & 7.74 & 11.25 \\
\hline bonded $\mathrm{S}=\mathrm{O}$ stretch & 1163 & 6.85 & 13.35 \\
\hline
\end{tabular}


Table 4. Vibrational frequencies $\left(\mathrm{cm}^{-1}\right)$, infrared (Debye $2 / \AA^{2}$.amu) and Raman $\left(\AA^{4 / a m u}\right)$ intensities of complexes of $\mathrm{MgSO}_{4}$ with three water molecules

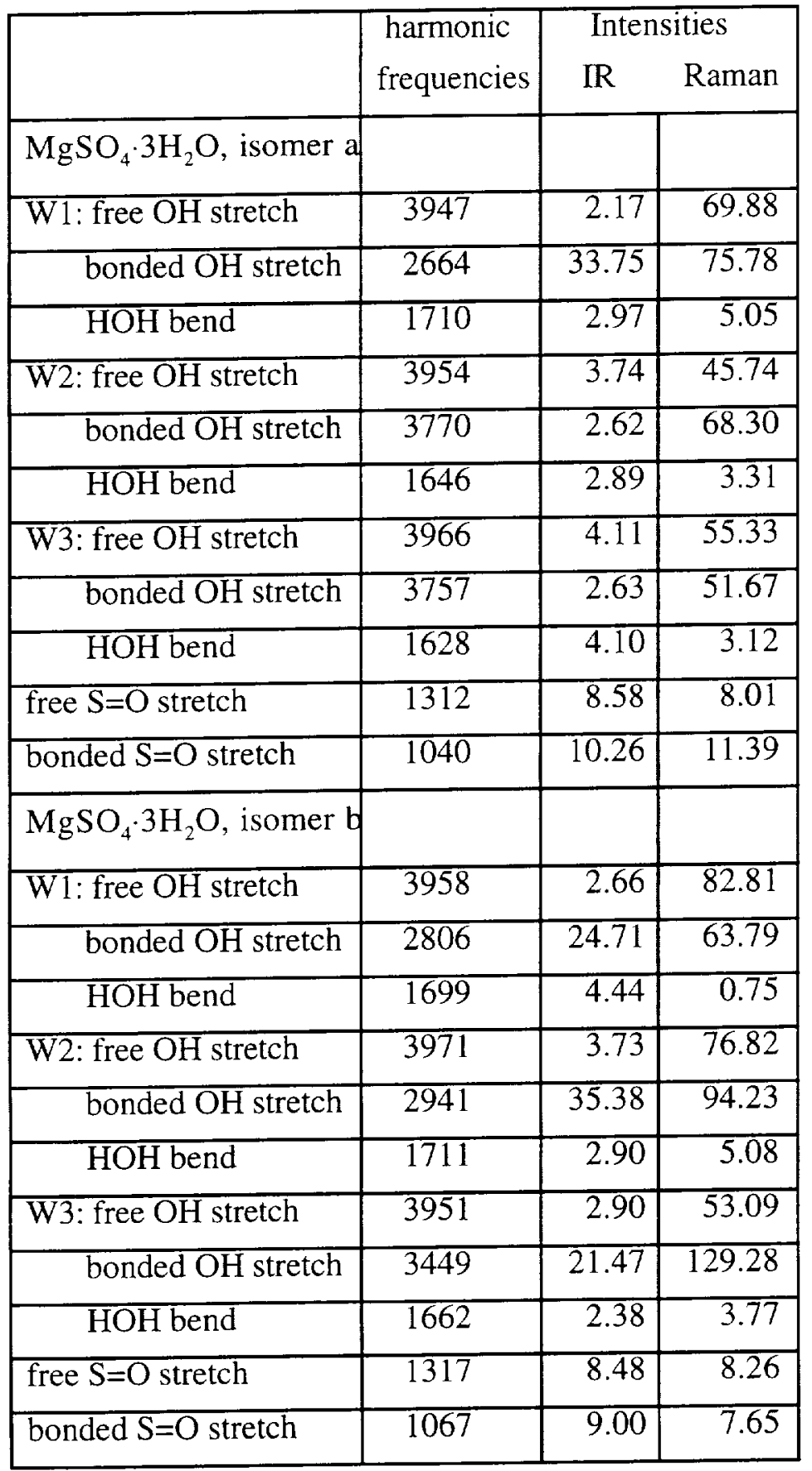



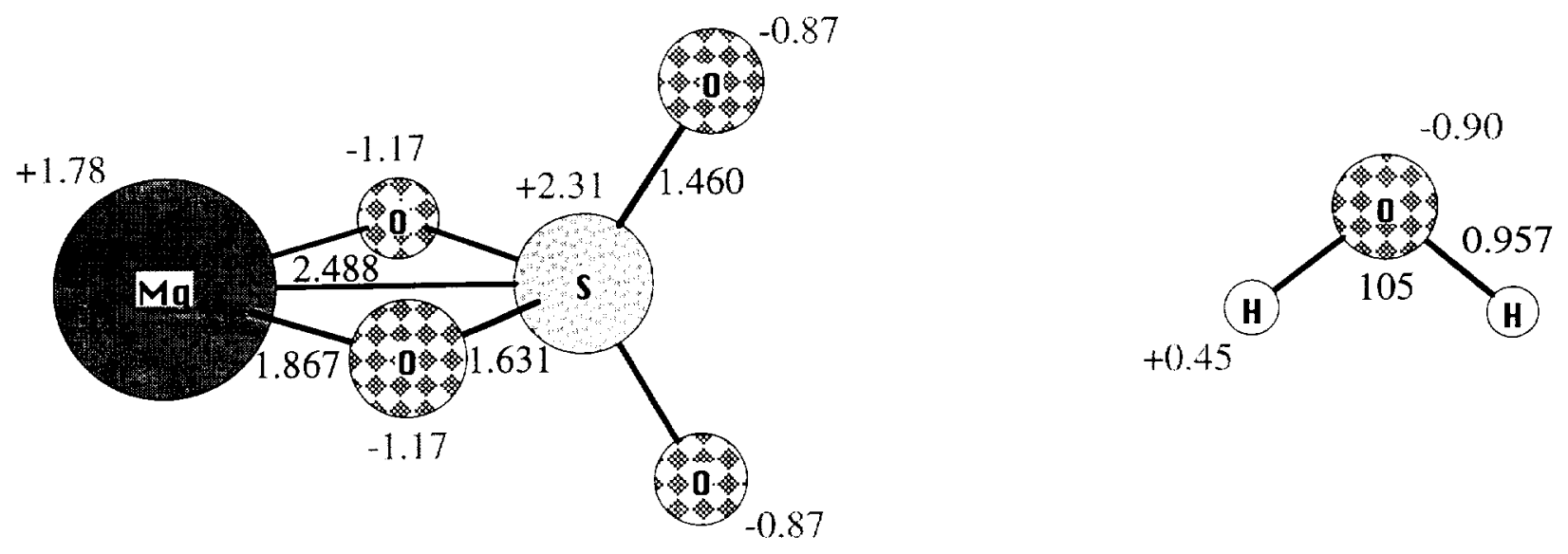

Figure 1. Equilibrium structures (angs.) and natural charges of pure $\mathrm{MgSO}_{4}$ and water molecules. 
(a)

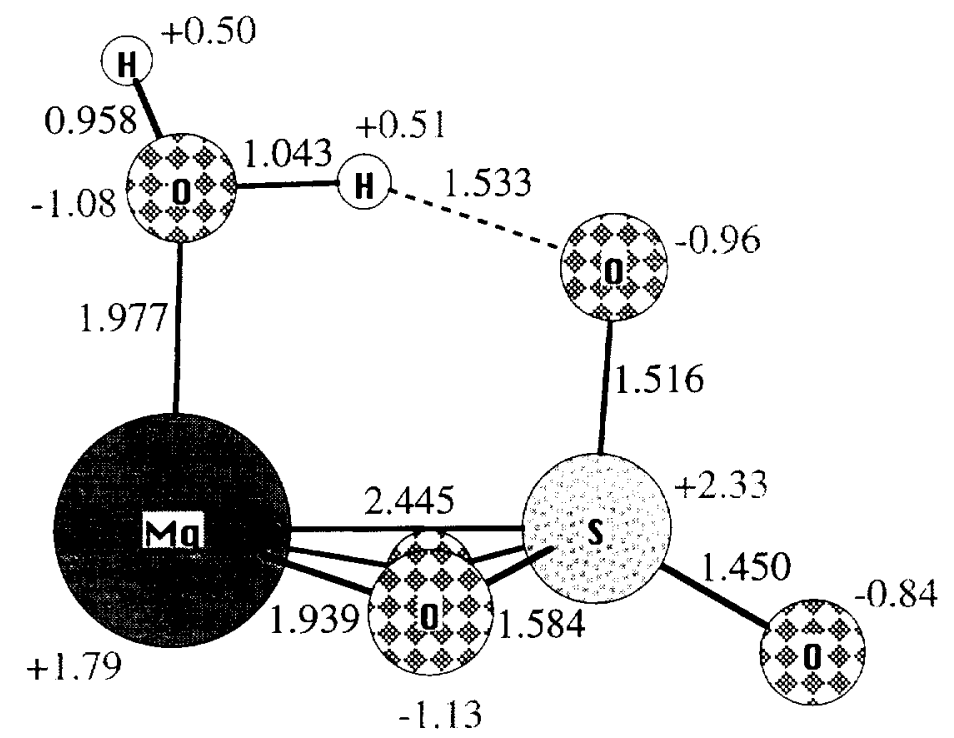

(b)

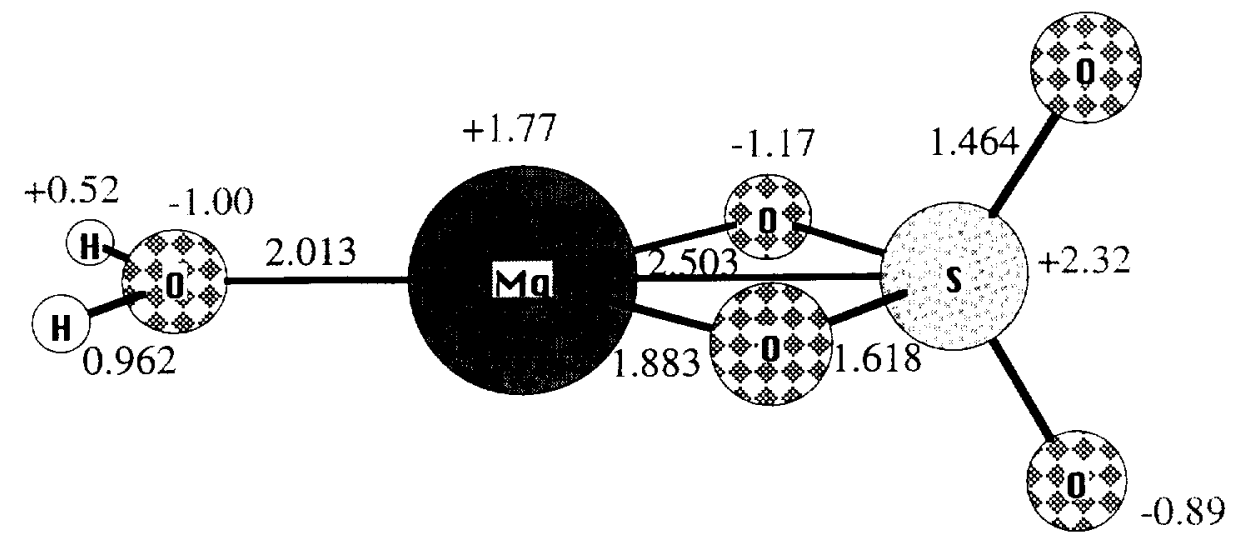

Figure 2. Equilibrium structures (angs.) and natural charges of complexes of $\mathrm{MgSO}_{4}$ with one water molecule. Structure (a) corresponds to the lowest energy isomer. 
(a)
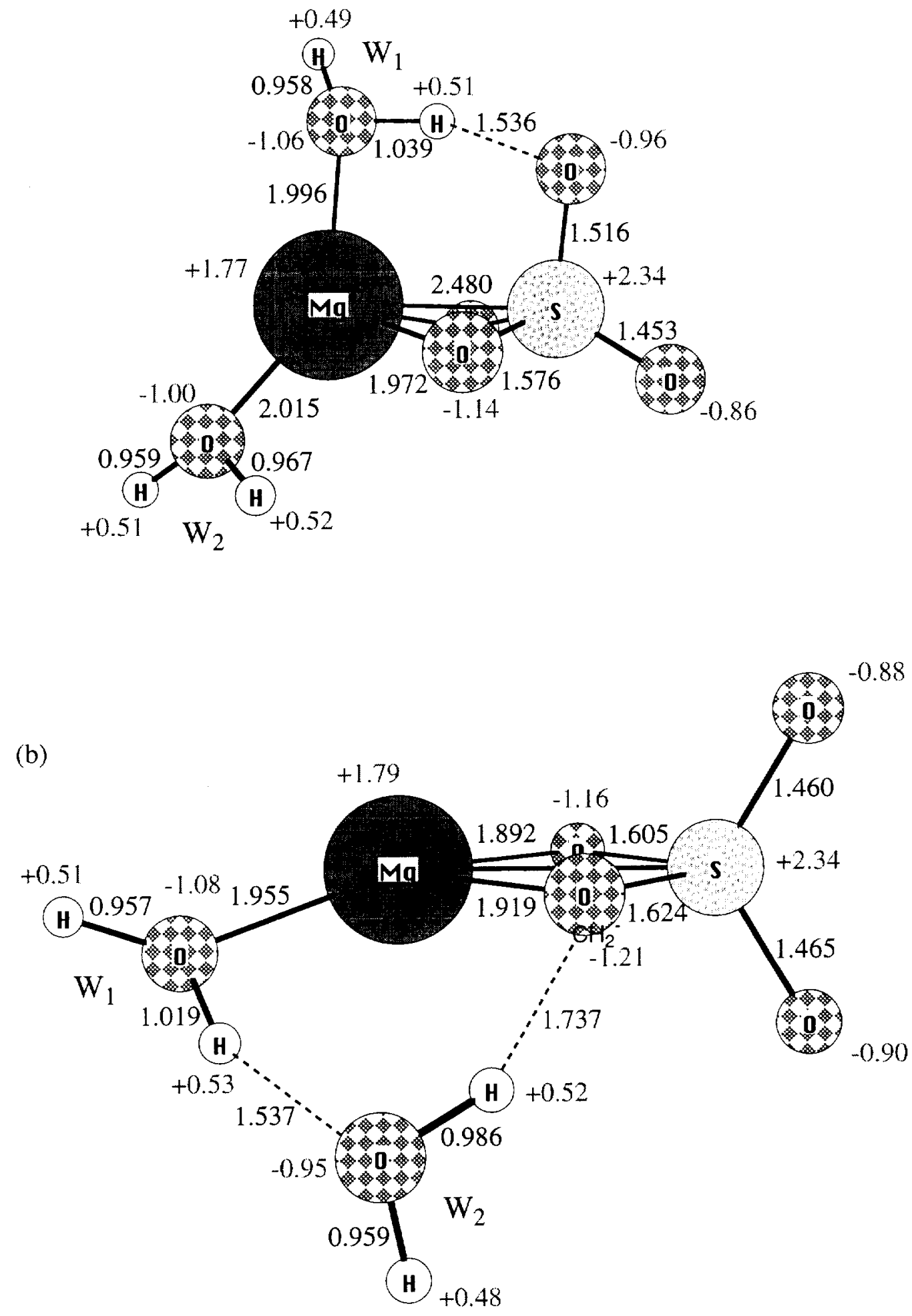

Figure 3. Equilibrium structures (angs.) and natural charges of complexes of $\mathrm{MgSO}_{4}$ with two water molecules. Structure (a) corresponds to the lowest energy isomer. 
(a)

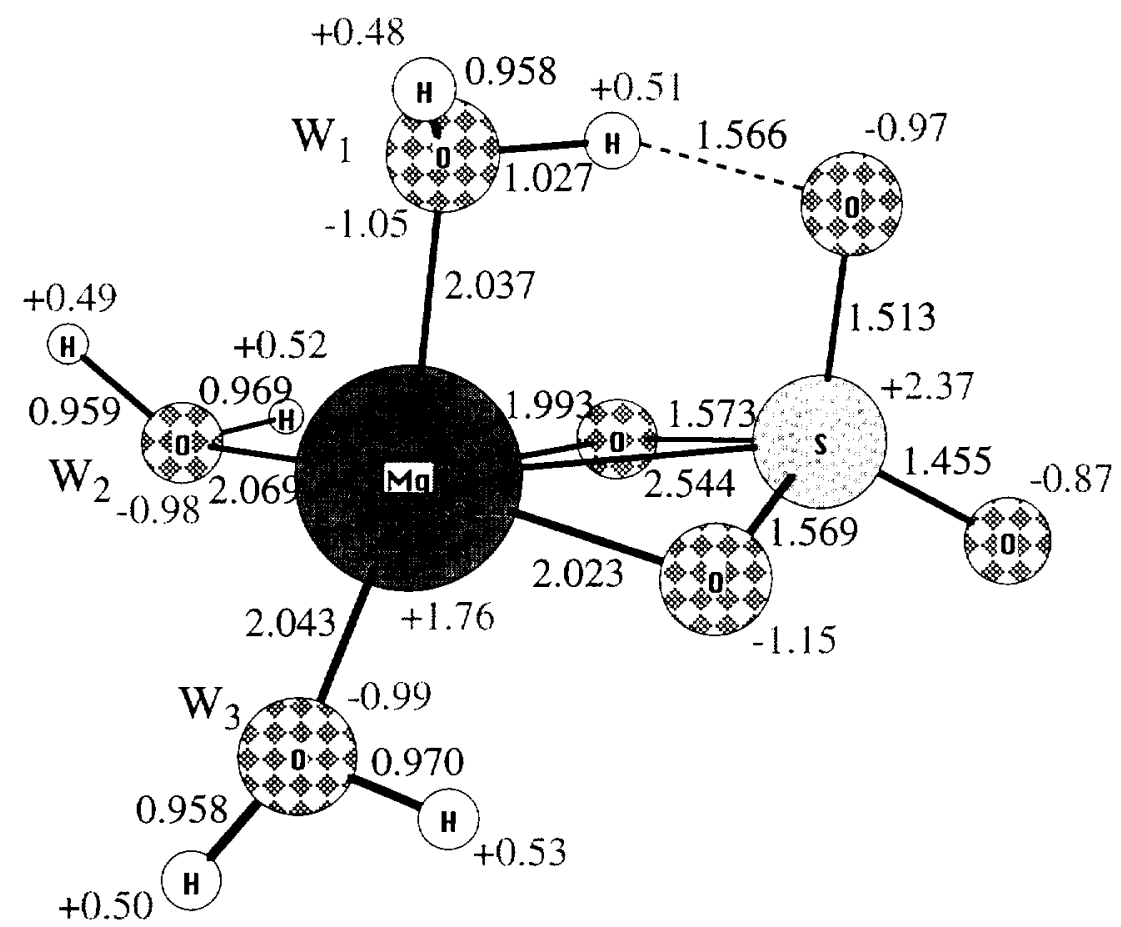

(b)

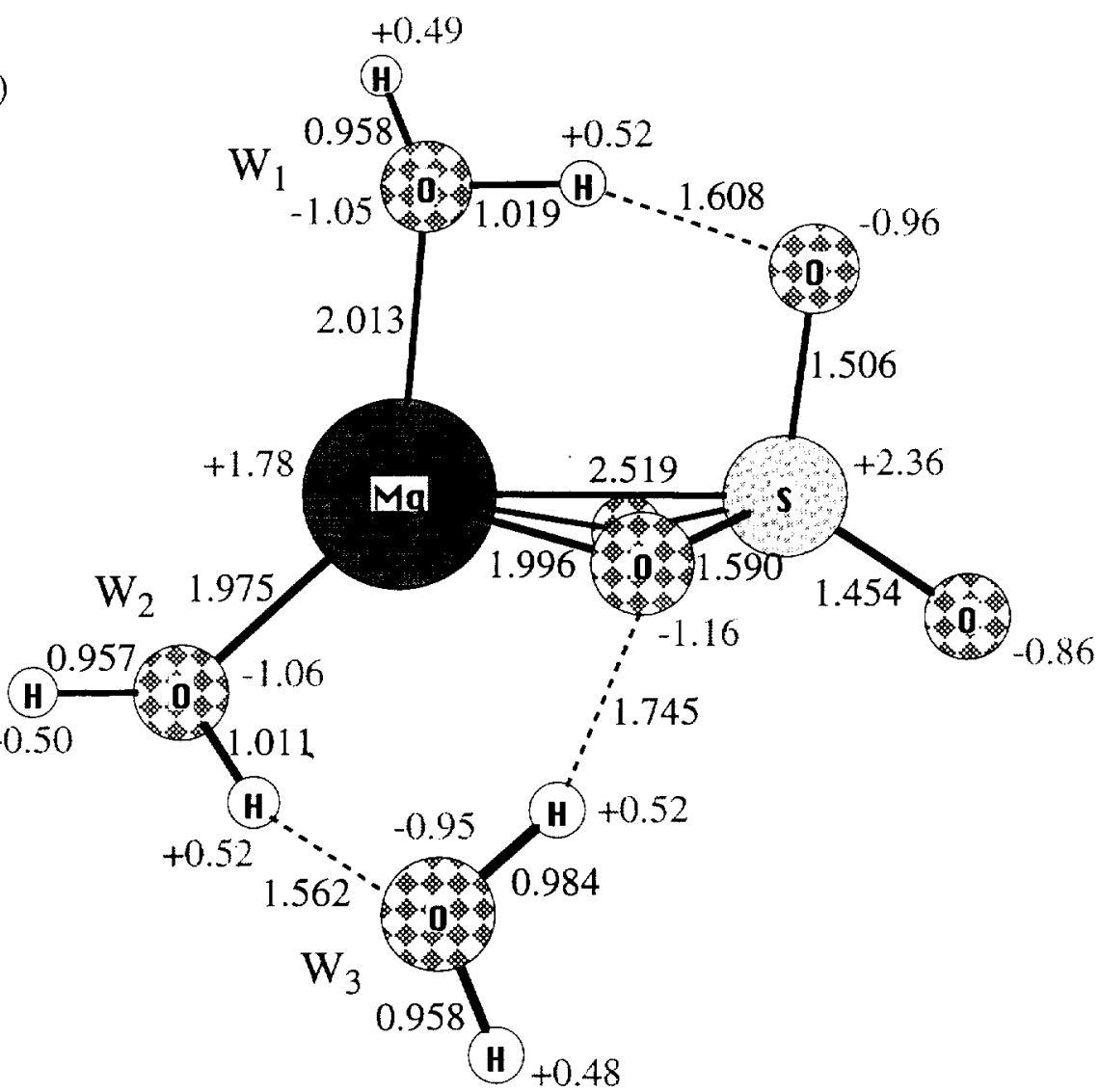

Figure 4. Equilibrium structures (angs.) and natural charges of complexes of $\mathrm{MgSO}_{4}$ with three water molecules. Structure (a) corresponds to the lowest energy isomer. 